\title{
Using computer conferencing to formulate a computer simulation of transitive behavior
}

\author{
ESTHER THORSON \\ Denison University, Granville, Ohio 49023 \\ and \\ TERRY F. BUSS \\ Ohio State University, Columbus, Ohio 43210
}

\begin{abstract}
The activities of a group engaged in simulation modeling via computer conferencing are described, and the model which resulted from those activities is briefly outlined. The phenomena which were modeled were initially described in a series of studies by Trabasso and his colleagues. These studies centered upon exhibition of transitive choices by children and adults, and supported the hypothesis that the choices were guided by formation of "linear arrays" of the choice objects.
\end{abstract}

There has been and continues to be extensive use made of computer simulations in psychology, both as teaching devices and as research tools. Models used as heuristic devices have been developed by, among others, Guetzkow (1962), Hermann and Hermann (1967), and Main (1974). Research simulations include those by Abelson and Carroll (1965), Colby (1964, 1967), Feigenbaum (1970), Newell and Simon (1961), Winograd (1973), and others.

A major difficulty in formulating such models is that wide and diverse sets of skills are required. To produce a computer simulation, the researcher must have a sophisticated understanding of the psychological process or phenomenon to be modeled and know the models that are available to account for that process or phenomenon. He must have a rudimentary understanding of programming languages, at least to the extent that he can give instructions to the programmer. Some might argue, in fact, that the greater the modeler's grasp of programming, the more successful will be his final product. Additionally, once the model exists as a computer program, its predicitons should be subjected to empirical testing in order to establish its validity or determine what constraints are operant in applying it to "real world" occurrences. Finally, if the model is to be used as a teaching device, it must be formulated in an interactive format so that students can easily and efficiently work with its empirical as well as its theoretical attributes.

In an effort to develop computerized models for research purposes while keeping in mind possible heuristic applications, the small group of psychologists introduced in preceding papers decided to choose an area of data and theory and work with each other through computer conferencing. The area selected concerned the problem of transitivity of choice in chil- dren and adults. However, because the group decided to focus on the experimental paradigm developed by Trabasso and his colleagues (e.g., Trabasso, Riley, \& Wilson, 1975) and because that paradigm apparently does not force children to reason transitively through sets of paired objects (e.g., if A is greater than B and if $B$ is greater than $C$, then $A$ is greater than $C$ ), the model that resulted was concerned with reasoning characterized by dependency upon a "linear array" of the choice objects.

\section{THE PROBLEM AREA: TRANSITIVITY IN CHOICE BEHAVIORS}

The transitive inference task has long been the focus of controversy in theories that attempted to describe various cognitive-developmental stages through which individuals pass from infancy to childhood. Piaget (1972) found that "pre-operational" children of ages less than 7 years could not perform transitive inferences over the lengths of three physical objects. He concluded, on the basis of clinical interview techniques, that this inability arises because of the child's failure to perform certain encoding and reversibility operations across pairs of objects. That is, children are able to encode the relations $A>B$ and $B>C$, but are unable to "reverse" the relation " $>$ " into " $<$ " with respect to $B$ in order to make the inference $A>C$. Piaget further theorized that transitive ability was manifested only after reaching the "concrete operational" stage, at approximately age 7 years.

Piaget's clinical interview technique is frequently criticized for measuring the child's verbal sophistication in discoursing about the transitive inference task, rather than the child's reasoning ability. To test Piaget's transitivity hypotheses in an experimental setting, Braine 
(1959) devised a procedure wherein nonverbal instructions and differential reinforcement were intended to control for verbal sophistication. Paired comparisons over the nonadjacent pair $(A, C)$ were made, and a third object $\mathrm{B}$ was introduced for comparison with $\mathrm{A}$ and $\mathrm{C}$. Braine found that the development of transitivity ability in children could, under this procedure, be lowered to ages 4 or 5 years. Smedslund $(1963,1969)$ questioned Braine's approach, suggesting that it was possible in Braine's paradigm to choose correctly B over A and C over B without performing any transitive inference operation, depending instead upon directly perceiving the differences between pairs. Smedslund attempted to eliminate this possibility by introducing an object $B$ for paired comparison with $\mathrm{A}$ and $\mathrm{C}$, but he made the longer object $\mathrm{A}$ always appear shorter and the shorter $\mathrm{C}$ always appear longer. Smedslund found that most children under 8 years of age were unable to behave transitively under these conditions.

The "Braine-Smedslund controversy" (Braine, 1964; Smedslund, 1963, 1969) generated further exploration of the transitivity problem in a series of studies by Bryant and Trabasso (1971), Riley and Trabasso (1974), Trabasso (1975), Trabasso and Riley (1975), and Trabasso, Riley, and Wilson (1975). Bryant and Trabasso (1971) first sought to eliminate perceptual illusions, such as those used by Smedslund, by associating a color with a particular stick of a given length so that after initial training, length was never observed. Presumably, then, symbolic relations were used rather than direct visual comparisons. Bryant and Trabasso also increased to five the number of objects compared. This allowed for measurement of the critical inferential pair $(B, D)$ in ordering $(A, B, C, D, E)$ which is derived by "reversing" alternative $C$ in pairs $(B, C)$ and $(C, D)$, respectively. Third, the researchers added an extensive training phase over the pairs $(A, B),(B, C),(C, D)$, and $(D, E)$ so that transitivity in responses could not be readily argued to result from lapse in memory. Finally, they asked the children to answer both questions in a pair, that is, "Which is longer, A or B?," "Which is shorter, A or B?" and so on. In this way, language sophistication, or the lack thereof, was presumably controlled, and the effects of "nominative" labeling were minimized. The results of these manipulations indicated the existence of suc- cessful transitive inference ability for children 4 to 6 years old.

Riley and Trabasso (1974) reexamined the Bryant and Trabasso (1971) study and attempted to refine the methodology. They modified the use of comparative questions for the presentation of the pairs, changed the spatial location of pairs being presented, and introduced differences between visual and verbal feedback. These changes, as well as the initial ones, yielded for Trabasso and his associates data that could be predicted by a number of different processing models and by variations in these models.

\section{MAJOR CHARACTERISTICS OF THE TRABASSO STUDIES}

All of the Trabasso transitivity studies (e.g., Trabasso, 1975) involved three conditions. Training Condition 1 included serial presentation, starting from either end of a series of lengths of pairs of differently colored sticks, and required that children associate colors with lengths so that they could respond correctly as to which of the pairs was longer without seeing the lengths. (Trabasso eventually used six sticks; only that case will be discussed here.) Generally, subjects in Training 1 learned five pairs quickly, averaging approximately .5 errors on each pair (Trabasso \& Riley, 1975).

Training Condition 2 involved presenting the same set of adjacent stick pairs, but in random order, and providing informational feedback until the subject reached a criterial accuracy level. Generally Training 2 produced a serial position curve in which more errors were made on the second and fourth pairs than on end pairs, and the third pair showed the greatest error rate. The pairs involving either the shortest or the longest sticks showed an "anchor effect" in that their error rate was always lower than pairs without either of the two sticks.

Trabasso's test condition involved presenting all 15 possible pairs of sticks and asking which stick of each pair was longer (or shorter). No feedback was given. Error rates and reaction times were examined, and typical results are shown in Table 1 . There are clear anchor effects. In addition, the pairs $(2,3),(3,4),(4,5)$, although showing slightly higher accuracy levels than

Table 1

Typical Reaction Time and Error Data From the Trabasso (1975) Test Conditions Compared With Predictions From the Distributional Model

\begin{tabular}{|c|c|c|c|c|}
\hline \multirow[b]{2}{*}{ Stick Pairs } & \multicolumn{2}{|c|}{ Percentage Correct } & \multicolumn{2}{|c|}{ Mean RT Rank } \\
\hline & Observed Mean & Predicted Rank & Observed & Predicted \\
\hline Both Anchors $(1,6)$ & 96.8 & 1 & 1 & 1 \\
\hline Short Anchors $(2,6)(3,6)(4,6)(5,6)$ & 94.0 & 3 & 3 & 3 \\
\hline Premises $(2,3)(3,4)(4,5)$ & 87.0 & 4 & 6 & 6 \\
\hline Inferences (one-step) $(2,4)(3,5)$ & 84.6 & 5 & 5 & 5 \\
\hline
\end{tabular}

* No comparable data points available 
"inference" pairs $(2,4),(3,5)$, and $(2,5)$, are responded to more slowly than the latter. In fact, comparison of adjacent nonanchored pairs, one-step inference pairs, and the two-step inference pair indicates decreased reaction times with increased "distances" between the sticks involved. This is opposite to what would be predicted by hypothesizing transitive inferences across component pairs. This outcome led Trabasso to suggest that subjects were arranging the stimulus lengths into "strings" or "linear arrays" and responding as a function of "distance" between the objects thus arrayed. It became clear that this problem no longer involved transitive inferences in the classical sense, but only transitive behavior. Many studies of differing design showed the same kinds of "linear array" results, and, in fact, models of the processing presumably occurring in Trabasso's test condition had been suggested. A brief description of some of these data, as well as the major models, follows.

\section{THE LINEAR ARRAY}

DeSoto and Bosley (1962) referenced early demonstrations of end anchoring (Volkmann, 1951) and noted that the serial position effect was described early by Deese (1958). DeSoto's own research indicated that when subjects were asked to associate various names as being freshmen, sophomores, juniors, or seniors, a serial position effect occurred. In a later study DeSoto, London, and Handel (1965) showed that subjects spontaneously ordered relational concepts like betterworse, lighter-darker, and above-below into a spatial array in a particular plane (e.g., horizontal, vertical). DeSoto et al, noted that "it can be argued that the inner procedure in thinking about orderings consists primarily in arranging elements on an axis in some cognitive space" (p. 520).

Murdock (1960) discussed the serial position effect in psychophysical tasks using physical rather than social stimuli, and formulated a mathematical theory which predicted the effect. For present purposes Murdock's "distinctiveness of stimuli" model can be said to assume that the distinctiveness of a stimulus depends upon the sum of its "distances" from every other stimulus in the set. The "relative percentage distinctiveness" then equals that sum of those distances divided by the total sums of distances of all the stimuli from each other. It can be shown that these two assumptions lead to predictions of the serial position effect (see Trabasso \& Riley, 1975).

Another mathematical approach to the same phenomenon is Bower's (1971) stimulus generalization model. This model assumes that the effectiveness of a stimulus depends upon its distinctiveness from a weighted-log definition of the adaptation level (AL). It also assumes that learning and generalization occur as in the Hull-Spence approach and, finally, that the probability of a response, $R_{j}$, to the presented stimulus depends upon the ratio of the strength of $R_{i}$ to that particular stimulus divided by the summed strengths of all other relevant responses to that stimulus (Luce's choice axiom). Trabasso and Riley (1975) applied Murdock's and Bower's models, at least indirectly, to the developmental data, arguing that both models showed reasonable fit and that for this application the two models are predictively isomorphic. Basically, each model will yield for each particular stick length the probability that each possible response will follow. These probabilities predict the serial position effect, and if probability is assumed to be inversely related to reaction time, they also predict accurately the rank order of reaction time for each stick pair.

Clearly, then, Trabasso's testing data could be approached by reference to existing mathematical models of the linear array. Unfortunately, these models had not been expanded to account for the original occurrences in the training conditions. The most reasonable suggestion for these conditions was made by Riley and Trabasso (1974), who hypothesized an "ends-inward" strategy for learning the pairs. Under this hypothesis the anchor pairs are acquired first, then the $(2,3)$ and $(4,5)$ pairs, and finally the center $(3,4)$ pair. There existed nowhere in the literature, however, a unified model that handled both training and testing.

\section{THE APPROACH OF THE CONFERENCING GROUP TO THE PROBLEM AREA}

This state of affairs, then, generally characterized the situation encountered by the conferencing group. Not only did group members think that a unified training and testing model would be of use to the research area, but there was consensus that the current work was not specific enough. Indeed, the group adhered to a position about modeling characterized by Newell's (1973) suggestion that "complete processing models rather than partial ones" (p. 300) are needed. Newell was arguing for a particular programming approach. Klahr and Wallace (1976), however, argued more gen. erally that "ultimately one hopes to push all such theorizing down to the concrete level of a running program" (p. 6). Since that goal was shared by members of the conference, the next step was to write flow diagrams for programs that would predict accurately in the data area.

Seven models, most of which examined only the test condition, were introduced via the computer conference. Each model was cast by its originator into a flow diagram format. Generally these models involved deterministic rather than probabilistic processes. Too, they assumed subjects performing the Trabasso task operated first on the longest, then on the shortest, sticks. Finally, most of the models involved a simple linear operator as the "learning" mechanism. 
Conference members individually worked through the models, criticizing, comparing, and revising them. By the time members met as a group, there was agreement that the enterprise was approaching a solution. During 3 days of further work, the group settled on a test model based on one of the diagrams introduced through the conferencing; the notion of probability distributions had to be added to the linear operator mechanism, and it was found not to be necessary to check long and short anchors first. The resulting test model stimulated Thompson and Spelt to formulate a training model that created the necessary probability distributions. When the meeting ended, outlines of the training and test processes had been formulated and some initial programming of the training condition had been started. It remained for the authors of this paper to work through the programming and structural changes that produced the current form of the model. ${ }^{1}$

\section{A COMPUTER SIMULATION OF ACQUIRING AND ACCESSING LINEAR ARRAYS}

Generally, the model underlying the computer simulation involves a process for developing generalization gradients corresponding to the various stick lengths and a process for accessing those distributions, generating appropriate reaction times. The simulation programs for cartying out the processes are currently operative, although some "fine tuning" and testing are still required. (The programs are written in BASIC + and copies can be obtained upon request from the first author.)

The training section of the program assumes that upon initial presentation the generalization gradients for each of the first two sticks $(1,2)$ are normal and have identical means and variances. For subsequent stick pairs, for example, $(2,3)$, three possible learning procedures were tested. Under the first, the stick not previously seen (3) is assumed to have a mean and variance equal to the stick with which it is paired (2). Under the second procedure, the new stick (3) is assumed to have a mean and variance identical to that of the first sticks $(1,2)$ before training. Both procedures assume the stick previously seen (2) maintains the mean and variance it acquired through its association with stick 1 . The third procedure, like the first, assumes the same starting means and variances for sticks 1 and 3 , but also assumes that both distributions for each stick (e.g., for stick 2, the distribution resulting from its being paired with stick 1 as well as that resulting from its pairing with stick 3 ) are retained, and later combined. The combined means are then simply the means of the means. The standard deviations, however, are calculated according to the formula:

$$
\sigma^{2}=\mathrm{p}\left(\sigma_{1}{ }^{2}+\mu_{1}{ }^{2}\right)+\mathrm{q}\left({\sigma_{2}}^{2}+\mu_{2}{ }^{2}\right)-\mu^{2},
$$

where $\mathrm{p}=\mathrm{q}=.5$ and $\mu=\mathrm{p} \mu_{1}+\mathrm{q} \mu_{2}$. In the succeeding part of the training program, whichever learning procedure is operative for sticks 1 and 2 then continues. Learning procedures result in predictions close to the Trabasso data, but the low error rate Trabasso found in Training 1 is best predicted by the third procedure. For that reason, only Procedure 3 will be referred to throughout the following.

In the training program, each time the subject responds to a training pair and is given feedback, the mean of the distribution for stick $1\left(\mu_{1}\right)$ is incremented by $a$ and $\mu_{2}$ is decreased by $b$. If the subject's response is correct, the standard deviation of the first distribution $\left(\sigma_{1}\right)$ is decreased by $\mathrm{c}$ and $\sigma_{2}$ is decreased by e. If the subject is incorrect, the standard deviations of the two distributions are increased by $d$ and $f$, respectively. The results are six distributions with serially ordered means, but with $\mu_{1}$ and $\mu_{6}$ further separated from the other means. (This is because the first and last sticks are paired only with one other stick; all the others are paired with two. This in turn means that $\mu_{1}$ is only incremented and $\mu_{6}$ is only decreased.)

The best fit for Trabasso's data appears to occur when $a=b, c>e$, and $d>f$. Under these circumstances the mean number of errors (or trials to criterion) across the five pairs is low and fairly equal. The standard deviations of distributions 1 and 6 are smaller than those of the other sticks, and the means are arranged in descending (or ascending) order, with $\mu_{1}$ and $\mu_{6}$ distributed further out from the central distributions. A typical set of values is shown in Table 2 .

During Training Condition 2, adjacent pairs are randomly sampled and feedback given. The simulation assumes on each trial, that the color of the stick "tags" a particular distribution; a sample observation is taken from that distribution, and the resulting value is com. pared to that value sampled from the other stick's distribution. The subject responds correctly if the sampled value of the larger stick is greater than that of the smaller stick. Otherwise he makes an error. The means and standard deviations change according to the

Table 2

Typical Parameter and Variable Values Resulting From the Distributional Model: Training Condition 1

\begin{tabular}{|c|c|c|c|c|c|c|c|c|c|c|c|c|c|c|c|c|c|c|c|c|c|}
\hline \multirow[b]{2}{*}{$\mu_{1}$} & \multirow[b]{2}{*}{$\sigma_{1}$} & \multirow[b]{2}{*}{$\mu_{2}$} & \multicolumn{4}{|c|}{ Initial Values } & \multirow[b]{2}{*}{$\mathrm{d}$} & \multirow[b]{2}{*}{ e } & \multirow[b]{2}{*}{ f } & \multicolumn{5}{|c|}{$\begin{array}{l}\text { Mean Errors for } \\
\text { Stick Pairs }\end{array}$} & \multicolumn{6}{|c|}{$\begin{array}{l}\text { Means and Standard Deviations } \\
\text { of Generalization Gradients }\end{array}$} & \\
\hline & & & $\sigma_{2}$ & $\mathbf{a}$ & $\mathbf{b}$ & $\cdot \mathrm{c}$ & & & & 1 & 2 & 3 & 4 & 5 & 1 & 2 & 3 & 4 & 5 & 6 & \\
\hline 1000 & 10 & 1000 & 10 & 1 & 1 & .1 & .1 & .05 & .05 & 3 & 1 & 0 & 1 & 1 & $\begin{array}{r}1013 \\
9.31\end{array}$ & $\begin{array}{r}988 \\
11.1\end{array}$ & $\begin{array}{r}980 \\
10.3\end{array}$ & $\begin{array}{r}970 \\
10.5\end{array}$ & $\begin{array}{r}959 \\
10.3\end{array}$ & $\begin{array}{r}941 \\
8.3\end{array}$ & $\begin{array}{l}\mu \\
\sigma\end{array}$ \\
\hline
\end{tabular}


same procedure used in Training 1 . The net result of Training 2 is to move the means of the distributions further apart and decrease the standard deviations. The end distributions remain further out.

During testing no further changes occur to the means and variances (since no feedback is given). As in Training 2, the subject samples distributions as a function of their color "tags," checks which sampled value is larger (or smaller), and responds accordingly. Errors are more likely for the central distributions since they exhibit greater overlap, and hence the appropriate serial position curves are generated. Reaction times are generated by multiplying a constant $\mathrm{C}$ by the absolute value of the reciprocal of the difference between the two observed values. This leads to accurate prediction of the reaction times for each pair type. A comparison of predicted and observed data points from the testing condition are shown in Table 1.

There are several advantages of the present model over prior models. First, the training process makes more intuitive sense than the ends-inward hypothesis. The question regarding the latter is how subjects can process end pairs "first" when, given the procedure used by Trabasso, subjects do not know what the final pair will be. The present model easily predicts the serial position results without having to assume that initial training order has any effect.

A second advantage of the model is that it integrates training and testing processes. Neither Murdock's model of stimulus distinctiveness nor Bower's stimulus generalization model have been applied to the training process, and hence these models must either assume equal means for each stick or assign means which, although isomorphic to those hypothesized here, are generated only arbitrarily and after the fact. The testing portion of the present model is identical in its predictions to Murdock's and Bower's, but it is more flexible in being able to respond to differences in training procedures. Furthermore, it directly generates reaction time predictions, a process that neither Murdock's nor Bower's model performs.

A third advantage stems from the fact that the model takes the form of a computer simulation, thereby specifying each component step of the hypothesized process. As in all such models, there are many possibilities for generating new hypotheses, for example, psychophysical testing of perceptions of the sticks' lengths to determine whether they do correspond to the hypothesized distributional forms. A gross way to do this involves asking for magnitude estimates of the stick lengths associated with each color. Another testable hypothesis is that the order of initial training has little or no influence on the occurrence of end anchor and serial position effects. This order can be easily manipulated. Manipulation of the relative lengths (or even diameters) of the sticks (Trabasso, Riley, \&
Wilson, 1975, performed one such manipulation) would be predicted to affect the location of the means of the distributions, and thereby affect both the resultant error rates and the reaction times. Finally, the model can be applied not only to judgmental tasks such as those described here, but also to tasks involving preference-based choices. In one such task, an analysis similar to Trabasso's showed outcomes consistent with those predicted by the present model (Buss, Thorson, \& Thorson, 1976).

We have emphasized the research aspects of a model developed through computer conferencing. This does not imply that extensive heuristic use cannot be made of the work accomplished through this project. One of the next steps, in fact, will be to package the model into an EXPER SIM (Stout, 1974) format. To the extent that the model is a useful research and heuristic tool, computer conferencing as an approach to modeling can be considered a viable and worthwhile methodology.

\section{REFERENCES}

Abelson, R. P., \& Carroll, J. D. Computer simulation of individual belief systems. American Behavioral Scientist, $1965,8,24-30$.

BowER, C. H. Adaptation-level coding of stimuli and serial position effects. In M. H. Appley (Ed.), Adaption-level theory. New York: Academic Press, 1971.

BraINE, M. D. The ontogeny of certain logical operations: Piaget's formulation examined by nonverbal methods. Psychological Monographs, 1959, 73(5, Whole No. 475).

Braine, M. D. Development of a grasp of transitivity of length: A reply to J. Smedslund. Child Development, 1964, 358, 779-810.

Bryant, P. E., \& Trabasso, T. Transitive inferences and memory in young children. Nature, 1971, 232, 456-458.

Buss, T. F., Thorson, E., \& Thorson, S. J. The transitivity of preferences and judgments in children. Report from the Polimetrics Laboratory, The Ohio State University, 1976.

CoLвy, K. M. Experimental treatment of neurotic computer programs. Archives of General Psychiatry, 1964, 10, 220-227.

ColBY, K. M. Computer simulation of change in personal belief systems. Behavioral Science, 1967, 12, 248-253.

DEESE, J. The psychology of learning. New York: McGrawHill, 1958.

DeSoto, C. B., \& Bosley, J. J. The cognitive structure of a social structure. Journal of Abnormal and Social Psychology, 1962, 64, 303-307.

DeSoto, C. B., London, M., \& HaNDel, S. Social reasoning and spatial paralogic. Journal of Personality and Social Psychology, 1965, 2, 513-521.

Feigendaum, E. A. Information processing and memory. In Norman, D. A. (Ed.), Models of human memory. New York: Academic Press, 1970.

GuETzxow, H. (Ed.). Simulation in the social sciences: Readings. Englewood Cliffs, N.J: Prentice-Hall, 1962.

Hermann, C. F., \& Hermann, M. B. An attempt to simulate the outbreak of World War 1. American Political Science Review, 1967, 61, 400-416.

KlAHR, D., \& Wallace, J. G. Cognitive development: An information-processing view. Hillsdale, N.J: Lawrence Erlbaum, 1976.

MaIN, D. (Ed.). Exper-sim on Michigan experimental 
simulation supervisor. Lexington, Mass: Xerox, 1974.

MurDock, B. B. The effectiveness of stimuli. Psychological Review. 1960, 67, 16-31.

Newell, A. You can't play 20 questions with nature and win. In W. G. Chase (Ed.), Visual information processing. New York: Academic Press, 1973.

Newell, A., \& Simon, H. Computer simulation in human thinking. Science, 1961, 134, 2011-2017.

PiageT, J. Judgment and reasoning in the child. Totawa, N.J: Littlefield, Adams, 1972.

RrLey, C. A., \& Trabasso, T. Comparatives, logical structures and encoding in a transitive inference task. Journal of Experimental Child Psychology, 1974, 17, 187-203.

SMEDSLUND, J. Development of concrete transitivity of length in children. Child Development, 1963, 34, 389-405.

Smedslund, J. Psychological diagnostics. Psychological Bulletin, 1969, 91, 237-248.

Stout, R. L. Modeling and the Michigan Experimental Simulation Supervisor: An overview and some prospects, Behavior Research Methods \& Instrumentation, 1974, 6, 121-123.

Trabasso, T. Representation, memory, and reasoning: How do we make transitive inferences? In A. D. Pick (Ed.), Minnesota symposia on child psychology (Vol. 9). Minneapolis: University of Minnesota Press, 1975.

Trabasso, T., \& Riley, C. A. On the construction and use of representations involving linear order. In R. L. Solso (Ed.), Information processing and cognition: The Loyolo symposium. Hillsdale, N.J: Lawrence Erlbaum, 1975.
Trabasso, T., Rirey, C. A., \& Wilson, E. G. The representation of linear order and spatial strategies in reasoning: A developmental study. In $R$. Falmagne (Ed.), Psychological studies of logic and its development. Hillsdale, N.J: Lawrence Erlbaum, 1975.

Volkmann, J. Scales of judgment and their implications for social psychology. In J. H. Rohrer \& M. Sherif (Eds.), Social psychology at the crossroads. New York: Harper, 1951.

Winograd, T. A procedural model of language understanding. In R. C. Shank \& K. M. Colby (Eds.), Computer models of thought and language. San Francisco: W. H. Freeman, 1973.

\section{NOTE}

1. Much of the conceptual work involved in the model outlined here derived from the group efforts of David Johnson (Albion College), Nancy Kirkland and Frank Ruggiero (Augustana College), Dana Main and John Breed (West Virginia College of Graduate Studies), Philip Spelt (Wabash College), Joseph Thompson (Washington and Lee University), and Rita Snyder (Denison University). The authors gratefully acknowledge the efforts and creativity of these individuals as well as those of Chris Vitale, who did most of the programming. We also thank the Lilly Endowment, Inc., for making the computer conferencing possible; John Ferguson of the Endowment for his guidance through the conferencing, and Thad Wilson and Robert Johansen of the Institute for the Future for arranging and aiding in the use of the conferencing system. 